\title{
CATEGORÍAS DE LAS NORMAS ESCOLARES
}

\section{CLASSIFICATION OF BEHAVIOUR NORMS IN SCHOOLS}

\author{
Cruz Pérez Pérez \\ Universidad de Valencia
}

\begin{abstract}
RESUMEN
En el presente artículo se propone una clasificación de las normas de comportamiento y convivencia que habitualmente se emplean en el medio escolar. Para ello se comienza analizando las principales clasificaciones de las normas existentes en la literatura sobre el tema, y se justifica la necesidad de realizar una nueva clasificación. El trabajo se inicia con la consulta, a través de un cuestionario, a un número significativo de profesores, y tras un proceso de análisis y concreción de estas aportaciones, se llega a una clasificación de las normas en seis grandes categorías y dieciséis subcategorías.
\end{abstract}

Palabras clave: clasificación, normas, comportamiento, medio escolar, categorías.

\begin{abstract}
In the present article, a classification of the behavior and living together norms, that are custonary used at school, is appointed. Departing of the previons classifications proposed by several authors, the need of accomplishing an own and new classification of that norms, is justified. For this, we toow advice of a significant number of school teachers and after sum up and analyse their contributions, we arribed to a classification of the scholastic norms in six large categories and seventeen sub-categories.
\end{abstract}

Key work: classification, norms, behavior, school, categories.

\section{Introducción}

La Reforma del Sistema Educativo concede una importancia fundamental al aprendizaje de los contenidos actitudinales, a los que eleva al mismo rango que los contenidos conceptuales y procedimentales, considerando que deben ser objeto de enseñanza, aprendizaje y evaluación. Los contenidos actitudinales incluyen el aprendizaje de actitudes, valores y normas. Sobre los dos primeros existen una gran variedad de estudios, clasificaciones y propuestas educativas, pero el análisis de las normas en el ámbito escolar, a pesar de su importancia educativa, apenas ha recibido la atención de los investigadores.

Una de las dificultades con las que se encuentra cualquier investigación sobre las normas escolares, es la falta de clasificaciones y categorizaciones válidas y adaptadas a los objetivos que se persiguen. 
El objetivo de este trabajo es el de establecer unas categorías en las que se puedan clasificar las normas que habitualmente se usan en el medio escolar, con la pretensión de que constituya un instrumento útil y valioso para llevar a cabo diversos análisis sobre las mismas: presencia y distribución de las normas en el aula, en el centro escolar, en las áreas de conocimiento, en las diferentes etapas educativas, comparación de las normas existentes en dos o más centros escolares, comparación de las normas empleadas por dos o más profesores, análisis de las normas existentes en el currículum escolar, en el proyecto educativo del centro, etc.

Sin un instrumento de este tipo, sería difícil analizar las normas existentes en cualquier contexto educativo, pues obtendríamos una larga lista en la que se mezclarían normas de todo tipo, y sería inviable el estudio de las mismas. Es necesario recurrir a algún tipo de clasificación que nos permita ordenarlas y valorar el modo como se distribuyen.

\section{Tipos de normas}

Si compleja era la definición del término norma o el análisis de sus componentes, a la hora de establecer los tipos de normas existen una gran variedad de agrupamientos o clasificaciones, según los criterios manejados por los autores que han tratado el tema. Por ello, más que enumerar las múltiples clasificaciones existentes, intentaré sintetizarlas en un único agrupamiento que describa las características fundamentales de los tipos de normas básicos. Estos serían los siguientes:

\section{Normas técnico-pragmáticas}

Estas normas contienen indicaciones, fundadas pragmáticamente, para el dominio de la naturaleza y la sociedad. Constituyen estándares generales fijados según puntos de vista de la finalidad, cuya determinación del contenido está elegida arbitrariamente, pero descansa en la convención.

Estas normas posibilitan una caracterización general, unitaria, de artículos de consumo y productos diversos, que por el hecho de estar normados, dan una información fiable sobre su medida. Algunos autores (Williams, 1975; Krings, 1978) incluyen en estas normas determinadas reglas para una conducta correcta. Así, por ejemplo, las reglas de tráfico son normas para una conducta recta en la conducción de vehículos, y las reglas del juego son normas para un juego correcto.

\section{Normas lógicas}

Determinan la forma consecuente y libre de contradicción. Su contenido es exclusivamente formal (causalidad, contradicción), y son condición necesaria de que algo, en general, pueda pensarse como objeto teórico. Lo que ha de pensarse como objeto, debe corresponder, necesariamente, a las leyes del pensamiento.

Puesto que la norma lógica es un concepto de relación, no tiene su sentido en sí misma; más bien sólo puede entenderse como norma en la «aplicación» en relación a aquello para lo que es norma. 


\section{Normas científicas}

Constituyen el marco para un sistema de enunciados verificables sobre un determinado ámbito de objetos. En analogía con la manera como las normas lógicas se hallan por la penetración reflexiva del saber de posibles objetos (experiencia), las normas científicas brotan de la construcción lógica de una determinada experiencia, que se hace en el contacto con la naturaleza, con lo psíquico, etc. A diferencia de las normas lógicas (substancia, causalidad), las normas científicas, logradas a base del enjuiciamiento de experiencias e intentos hipotéticos, son conceptos menos abstractos, por poseer mayor contenido; estos contienen especiales determinaciones reales, que no valen para todo ámbito de experiencia, sino para uno determinado y delimitado. Sin embargo, normas lógicas y científicas tienen la misma función: fundamentar un saber y abrirlo a su verdad.

\section{Normas prácticas}

Bajo este tipo de normas se agrupan los principios jurídicos, religiosos o morales que incitan al seguimiento de fines o a ejecutar acciones.

Normas morales: Su campo de actuación es el de la conciencia, es decir, el de la intimidad del sujeto. Estas normas abarcan enteramente la vida de todo individuo, sin prescindir de ninguno de sus factores y aspectos, y enfocándola en términos absolutos. La norma moral orienta al individuo hacia su cumplimiento, porque esto constituye la realización de un valor en la vida. Supone y requiere libertad en su cumplimiento, pues para que una conducta pueda ser objeto de un juicio moral, es preciso que el sujeto la realice por sí mismo, que responda a una posición de su propio querer.

Normas jurídicas: Son aquellas que instituyen los derechos y deberes de cada miembro perteneciente a una sociedad, y señalan las funciones o «roles» que cada uno debe desempeñar en su comunidad. La norma jurídica trata de crear el orden social, el orden de las relaciones objetivas entre los individuos, el de las vinculaciones entre estos; en suma, el orden de las estructuras colectivas, el orden del tejido en que se mezclan y condicionan mutuamente, de modo objetivo, las conductas de varios sujetos. La diferencia fundamental que presentan estas reglas respecto a las demás, reside en su carácter obligatorio para los sujetos. Son normas formuladas por el Estado -aunque en sociedades más primitivas no se daba esta circunstancia- y sostenidas por la fuerza moral y coercitiva del mismo.

Normas religiosas: Parten de principios religiosos e incitan al seguimiento de determinados fines congruentes con ellos. Suelen constituir una mezcla diversa de reglas, a veces incluso mezcladas y confundidas con normas jurídicas.

Tras esta clasificación de síntesis realizada, considero necesario exponer la que propone Von Wright, por lo global y clarificadora que resulta de algunos aspectos de las normas. Von Wright (1970, p. 21 y ss.) distingue entre tres grandes grupos o tipos de normas: reglas, prescripciones y directrices. En adición a estos tres grandes grupos, señala tres grupos menores de particular importancia: las costumbres, los principios morales y las reglas ideales. 


\section{Las reglas del juego}

Las reglas del juego son el prototipo y ejemplo clásico de un tipo importante de norma. La actividad del juego se desarrolla con arreglo a patrones fijos que se pueden llamar movimientos del juego. Las reglas del juego determinan esos movimientos o patrones y de esa forma, también el juego mismo y la actividad de jugarlo. Su grado de flexibilidad y mutabilidad es muy escaso.

\section{Las prescripciones}

Las prescripciones son dadas o dictadas por alguien. Tienen su origen en la voluntad de un dador de normas (autoridad normativa). Van, además, destinadas o dirigidas a algún agente o agentes (sujetos normativos). La autoridad que da la norma, normalmente, quiere que el sujeto(s) adopte una cierta conducta, que se comporte de una determinada manera. La autoridad promulga la norma para que el sujeto(s) conozca su voluntad, y para darle efectividad añade una sanción, amenaza o castigo a la norma. Todos estos aspectos diferencian a las prescripciones de las reglas.

\section{Las costumbres}

Las costumbres, en algunos de sus aspectos son como reglas y en otros como prescripciones. Se pueden considerar como una especie de hábitos. Un hábito, es primordialmente, una regularidad en la conducta de un individuo, una disposición o tendencia a hacer cosas similares en ocasiones similares. Los hábitos se adquieren; no son innatos. Las costumbres se pueden considerar como hábitos sociales. Son patrones de conducta para los miembros de una comunidad, que se van adquiriendo a lo largo de su historia, y son, más bien, impuestos a sus miembros que adquiridos por éstos individualmente. Las costumbres influyen sobre la conducta ejerciendo una «presión normativa» sobre cada uno de los miembros de la comunidad. La existencia de esta presión se refleja en las diversas medidas punitivas con las que la comunidad reacciona ante aquellos de sus miembros que no se ajustan a sus costumbres. En este sentido se asemejan a las normas como prescripciones, pero hay también importantes diferencias respecto a las mismas. Ante todo, las costumbres no les son dadas a los sujetos por autoridad alguna que no sea la propia comunidad, incluyendo tanto los miembros pasados como los presentes.

Otra diferencia entre las costumbres y las prescripciones es que las primeras no necesitan promulgación por medio de signos simbólicos, es decir, que no tienen que estar escritas en ninguna parte. Por ello podrían llamarse también «prescripciones implícitas».

\section{Directrices o normas técnicas}

Se refieren a los medios a emplear para alcanzar un determinado fin. El ejemplo más claro de normas técnicas son las «instrucciones para el uso», con las que se supone que la persona que las sigue aspira a la consecución de un fin o resultado.

La conceptualización que realiza Von Wright de este tipo de normas, coincide, básicamente, con lo expuesto anteriormente sobre las normas técnicas-pragmáticas. 


\section{Las normas morales}

En torno a la naturaleza de los principios morales ha habido mucha controversia y desacuerdo. Algunos filósofos los consideran como una especie de prescripción (mandamientos o leyes de Dios a los hombres). La leyes del estado tienen, frecuentemente, un «contenido moral» o están relacionadas con materias morales. Lo mismo ocurre con las prescripciones que los padres imponen a la conducta de sus hijos. En la vida moral del hombre, las prescripciones desempeñan un papel prominente, lo cual es una característica lógica de la moralidad. Pero este vínculo lógico de las normas morales y las prescripciones, no puede reducir las primeras a una especie de las segundas.

También existe una relación del aspecto prescriptivo de la moralidad con las costumbres, como por ejemplo en las ideas morales en materias relativas a la vida sexual.

La segunda alternativa que se da en la historia de la ética, es una visión teleológica de la moralidad, según la cual, las normas morales son un especie de normas o directrices técnicas para el logro de ciertos fines. Pero ¿qué fines? ¿La felicidad del individuo? ¿El bienestar de la comunidad?

Tanto la concepción de las normas morales como prescripciones, como la concepción teleológica de las mismas, tropieza con serias dificultades, lo cual, ha llevado a sugerir que las normas morales son «conceptualmente autónomas»; un grupo de normas que se mantiene por sí solas. Esta consideración de las normas morales se conoce con el nombre de posición «deontológica».

Von Wright realiza una crítica al deontologismo en la ética, considerándolo una vía de escape ante las dificultades y una posición totalmente insatisfactoria:

«La peculiaridad de las normas morales, tal como yo las veo, no está en que formen un grupo autónomo por sí solas; está más bien en que tienen complicadas afinidades lógicas con los otros tipos principales de normas y con las nociones valorativas de bien y mal. Comprender la naturaleza de las normas morales no es por eso descubrir una única característica de ellas; es examinar sus complejas afinidades con cierto número de otras cosas». (Von Wright 1970, p. 32).

\section{Reglas ideales}

De acuerdo con G.E. Moore, Von Wright llama así a las normas que tienen más relación con ser que con hacer. Hacemos referencia a estas reglas cuando, por ejemplo, decimos que un hombre tiene que ser generoso, sincero, justo, ecuánime, etc. Las reglas ideales están en estrecha relación con el concepto de bondad. En este sentido, es útil distinguir entre los principios morales, que son normas de acción moral, y los ideales morales, que establecen el patrón de hombre bueno.

Se puede considerar que las reglas ideales mantienen una posición intermedia entre las normas técnicas acerca de los medios para un fin y las reglas que determinan un patrón o modelo.

Finalmente, tras las clasificaciones expuestas, hay que tener en cuenta que cuando hablamos de «saber acerca de las normas», nos referimos fundamentalmente a los «saberes prácticos», que son aquellos que pretenden dirigir o influir en el obrar humano, y pueden 
ser morales, jurídicos, técnicos, religiosos, etc. y se denominan prácticas, a las disciplinas que se ocupan de ellos.

\section{La clasificación de las normas}

Realizando una exploración por la bibliografía existente sobre el tema, encontramos algunas clasificaciones de normas, pero la mayoría están referidas a las normas en general, y no a las normas en el ámbito escolar. Las clasificaciones de normas más importantes, que se pueden encontrar en los trabajos de las últimas décadas, son las siguientes

- Parsons (1951) establece seis focos principales en la regulación normativa de la conducta humana:

- El funcionamiento interno de la personalidad individual.

- Las relaciones con el mundo de los fenómenos físicos y biológicos externos al sujeto.

- Las relaciones entre personas y entre grupos dentro de la sociedad.

- Las relaciones entre sociedades.

- La integración intracultural.

- Las relaciones del hombre con los entes y sucesos sobrenaturales.

- Pepper (1960), centrándose en el área de actividad y de experiencia humana a la que las normas se refieren, las clasifica en:

- Normas técnicas

- Normas hedonistas

- Normas integradoras de la personalidad

- Normas sociales

- Normas institucionales

- Von Wright (1979) las clasifica en tres grandes grupos:

- Reglas: del juego, de la gramática, de la lógica, de las matemáticas, etc.

- Prescripciones: mandatos, permisos, prohibiciones.

- Directrices: normas técnicas.

- Gibbs (1985), distingue entre costumbres, mores, reglas y leyes.

- C. Gotzens (1986), en el ámbito escolar, y centrándose en el comportamiento del alumno, realiza la siguiente clasificación:

- Normas referidas al estudio de los alumnos

- Normas referidas a la responsabilidad social del alumno

- Normas que regulan las relaciones sociales en clase.

- Normas que regulan los comportamientos asociados a la inmadurez orgánica del individuo (hiperactividad, distracción, holgazanería...)

- Schmuck y Schmuck (1974), clasifican las normas en cuatro grandes grupos:

a) Normas perceptuales: Se refieren a la capacidad de compartir el modo de percibir del grupo.

b) Normas cognoscitivas: Se refieren a los procesos de pensamiento como razonar, recordar, analizar, calcular. Se dividen en dos grandes categorías: 
- Normas cognoscitivas como metas de la clase o grupo

- Normas cognoscitivas sobre el aprendizaje en el aula

- Normas referentes a los contenidos de la enseñanza

- Normas referentes a los procedimientos

c) Normas evaluativas: Hacen referencia a lo que es bueno o malo, conveniente o inconveniente, etc. Involucran grandes cantidades de sentimientos positivos y negativos.

d) Normas de conducta: Las referidas al comportamiento del alumno en el grupo.

- Curwin (1983), establece las siguientes categorías de normas, con el objeto de realizar una lista de normas que afecten al profesor o a los alumnos:

a) Realización de tareas y planes de clase

b) Obediencia al profesor

c) Toma de decisiones para las que no existen reglas

d) Uso de frases hirientes (humillación, sarcasmo)

e) Mostrar enfado

f) Mostrar miedo

g) Mostrar tristeza

h) Mostrar cariño

i) Cuidar las cosas de la escuela

j) Movimiento en la clase

- Turiel (1984), en una investigación sobre los argumentos que utilizan los niños para justificar los juicios sobre las reglas, las clasifica en cuatro categorías distintas:

a) Bienestar y justicia

b) Estructura social (costumbre, tradición, autoridad, coordinación social)

c) Proceso (reglas sobre el trabajo y actividades de aprendizaje)

d) Otras entre las que se incluye la prudencia y la elección personal.

Las clasificaciones revisadas responden a criterios muy dispares, debido a que el concepto de norma abarca un campo de significado muy amplio y heterogéneo. Aquellas que más se aproximan al concepto de norma en el ámbito escolar, son las propuestas por Curwin, Hargreaves y el grupo $4^{\circ}$ de Schmuck y Schmuck sobre normas de conducta. Sin embargo, ninguna de estas clasificaciones, por sí sola, abarca todas las normas del ámbito escolar, debido a lo cual opto por extraer de cada una de ellas aquellos aspectos que considero válidos para realizar una nueva clasificación. Esta tarea la abordo recabando la ayuda de un número significativo de profesores y profesoras de diversos centros escolares.

\section{Descripción de la experiencia}

Se trata de realizar una clasificación y categorización de las principales normas que habitualmente se emplean en el medio escolar. Para ello se recaba la colaboración de un total de 106 profesores, pertenecientes a cinco colegios públicos de la ciudad de Valencia y su entorno, que son los siguientes: 


\author{
Colegio \\ C.P. Castellar-Oliveral \\ C.P. Horno de Acedo \\ C.P. Colon \\ C.P. García Planells \\ C.P. Pablo Neruda
}

$\begin{array}{lc}\text { Población } & \mathbf{n}^{\mathbf{0}} \text { de profesores } \\ \text { Castellar } & 34 \\ \text { Horno de Acedo } & 12 \\ \text { Benetuser } & 23 \\ \text { Manises } & 23 \\ \text { Valencia } & 34\end{array}$

A los profesores de dichos centros se les presenta el cuestionario que figura en el Anexo I, en el cual figura una clasificación de las normas escolares en categorías y subcategorías, y se les pide que lo analicen y valoren la clasificación realizada utilizando criterios de adecuación a la realidad escolar.

\section{Análisis de los resultados}

El cuestionario fue cumplimentado por 56 profesores. El dato más significativo de las aportaciones realizadas, es que 48 profesores (el 86\%) consideran que las categorías establecidas son básicamente apropiadas para clasificar las normas del aula y del centro. Entre las sugerencias más importantes, extraídas de las respuestas de los profesores, se encuentran:

- Incluir el respeto a las personas en el mismo nivel que la convivencia en el aula y de un modo diferenciado ( $19 \%$ de los profesores).

- La inclusión del cuidado de los espacios comunes del centro (patio, pasillos, servicios, laboratorio, etc.) en la misma categoría del cuidado de materiales $(12 \%$ de los profesores).

- La reformulación de la categoría propuesta como «salidas del centro» a la denominación, más precisa de actividades complementarias, comportamiento cívico o comportamiento como ciudadanos en el ámbito extra-escolar (17\% de los profesores).

- Establecimiento de una nueva categoría de normas que abarque todo lo relacionado con la salud, la higiene, hábitos de limpieza, el cuidado del cuerpo, etc. (27\% de los profesores). Las subcategorías de normas que proponen son muy variadas y adoptan diversas denominaciones.

\section{Conclusiones y propuestas}

En un esfuerzo de síntesis de todas las aportaciones que realizan los profesores, he establecido unos subgrupos o subcategorías, los cuales, debido a la interrelación existente entre las normas que los integran, resultan en ocasiones difíciles de diferenciar. Sin embargo, he considerado necesario y útil llegar a este nivel de diferenciación, pues ello va a permitir realizar un análisis más pormenorizado de las normas que incluyen. Así pues, tras la incorporación de las aportaciones realizadas por los profesores encuestados, establezco la propuesta de categorías y subcategorías de normas que figura en el punto siguiente: 


\begin{tabular}{|c|c|}
\hline Categorías de normas & Subcategorías de normas \\
\hline $\begin{array}{l}\text { Normas referidas al funcionamiento } \\
\text { democrático en el aula. }\end{array}$ & $\begin{array}{l}\text { a) Participación en el establecimiento de } \\
\text { normas } \\
\text { b) Participación en asambleas, debates, etc. } \\
\text { c) Respeto a las decisiones democráticas. }\end{array}$ \\
\hline $\begin{array}{l}\text { Normas sobre el respeto a las personas y a } \\
\text { la convivencia en el aula y en el centro. }\end{array}$ & $\begin{array}{l}\text { a) Respeto a los derechos de los demás. } \\
\text { b) Respeto a la diversidad, no discriminación. } \\
\text { c) Organización y relaciones entre alumnos. }\end{array}$ \\
\hline $\begin{array}{l}\text { Normas sobre el cuidado y utilización de los } \\
\text { materiales del aula y espacios comunes del } \\
\text { centro. }\end{array}$ & $\begin{array}{l}\text { a) Materiales y espacios } \\
\text { b) Espacios comunes }\end{array}$ \\
\hline Normas sobre el trabajo en el aula. & $\begin{array}{l}\text { a) Trabajos individuales } \\
\text { b) Trabajos en equipo } \\
\text { c) Actividades en gran grupo } \\
\text { d) Cumplimiento de responsabilidades }\end{array}$ \\
\hline Normas sobre el cuidado de la salud. & $\begin{array}{l}\text { a) Cuidado del cuerpo } \\
\text { b) Higiene y aseo personal }\end{array}$ \\
\hline $\begin{array}{l}\text { Normas sobre el comportamiento como } \\
\text { ciudadanos en el ámbito extraescolar. }\end{array}$ & $\begin{array}{l}\text { a) Recursos y espacios naturales } \\
\text { b) Lugares públicos } \\
\text { c) Patrimonio histórico-artístico }\end{array}$ \\
\hline
\end{tabular}

El contenido y justificación de las categorías y subcategorías de normas establecidas se desarrolla en los siguientes puntos.

\section{Normas referidas al funcionamiento democrático en el aula}

Abarca todas aquellas normas que hacen referencia al funcionamiento democrático del aula y de algunos aspectos del centro.

En una sociedad democrática es fundamental que los alumnos aprendan, de una manera «vivenciada», los mecanismos por los que ésta se rige, al objeto de que puedan asumir los valores del sistema como propios.

El Sistema Escolar, de un modo lento pero progresivo, va descartando los modos de relación profesor-alumno jerarquizados, distantes y autoritarios, para dar paso a unas relaciones más abiertas, igualitarias y participativas, acordes con los valores democráticos de la sociedad en que vivimos. Regular este modo de convivencia, implica la existencia de un conjunto de normas que constituyen una categoría con entidad propia.

Se han establecido las subcategorías Participación de los alumnos en la elaboración de las normas, y Respeto a las decisiones tomadas democráticamente para poder analizarlas de una manera diferenciada a la realización de asambleas, debates, discusión de problemas, etc., La razón para esta diferenciación, está en que estos aspectos los considero «clave» en el aprendizaje de los principios democráticos, y pienso que requieren mecanismos de aprendizaje específicos. 


\section{a) Participación en el establecimiento de normas}

Realización de propuestas de normas, análisis de las mismas, votaciones, etc. Establecimiento de turnos, responsabilidades concretas sobre el cumplimiento de las normas y en general, cualquier modo de colaboración en la organización social de la comunidad educativa.

\section{b) Participación en asambleas, debates, etc.}

Participación responsable en cualquier modalidad de diálogo, discusión, toma de decisiones, resolución de conflictos. Aportación de opiniones propias y respeto hacia las de los demás.

Si en cualquier grupo social es necesario regular la intervención de las personas que participan en esas actividades, en el medio escolar se hace imprescindible establecer unas normas claras y concretas de participación, para que los alumnos aprendan a respetar los turnos de palabra, realizar las intervenciones necesarias, ajustarse a un tiempo, mantener posturas flexibles para llegar a un consenso, etc.

\section{c) Respeto a las decisiones tomadas democráticamente}

Conjunto de normas que regulan el respeto y cumplimiento de las decisiones tomadas de modo democrático.

Cuando no existen estas normas, así como unas consecuencias para sancionar su infracción, las decisiones tomadas por la mayoría va perdiendo fuerza y acaban siendo inviables de llevar a la práctica, lo cual deteriora la imagen del sistema democrático. Por ello, es necesaria la existencia de un conjunto de normas que obligue a los alumnos a respetar los resultados de las votaciones, las reglas de intervención, las sanciones acordadas y, en general, todas aquellas decisiones establecidas por la mayoría.

\section{Respeto a las personas y a la convivencia en el aula y el centro}

Hacen referencia a todas aquellas situaciones y conflictos que genera la convivencia e interacción de un grupo de personas en el aula y centro.

Constituyen el núcleo básico de normas de comportamiento que regulan habitualmente la vida colectiva en el aula y el centro. Este conjunto de normas puede ser más o menos amplio, y de ellas se puede deducir el talante más o menos democrático y abierto de una comunidad educativa, según el modo como se aborde el respeto a las personas o las situaciones conflictivas que genera la convivencia diaria.

Se resalta el respeto a los derechos de los demás, como una subcategoría para el análisis de las normas, porque constituye uno de los valores fundamentales que se deben preservar en todo momento, y constituyen la base de cualquier organización de la convivencia. La subcategoría «Respeto a la diversidad, no discriminación», que se incluiría en la anterior, se ha diferenciado por la importancia que tiene para nuestra sociedad, y para aquellos alumnos que se consideran «diferentes», el mantenimiento de actitudes de tolerancia.

Las subcategorías de normas establecidas son las siguientes: 


\section{a) Respeto a los derechos de los demás}

Todo alumno, como persona, tiene una serie de derechos que se deben garantizar (dignidad, intimidad, propiedad, etc.). Constituyen un conjunto de normas que regulan el respeto a los derechos humanos, los cuales son básicos para garantizar la justicia en el aula y la igualdad de las personas que la integran.

\section{b) Respeto a la diversidad, no discriminación}

Desigualdades sociales provocadas por el lugar de nacimiento, residencia, raza, sexo, condición económica, cultura a la que se pertenece, etc.

$\mathrm{Al}$ igual que ocurre en cualquier sociedad, el nivel moral de la misma se puede medir por la forma como se trata a las personas diferentes. Los alumnos aprenden actitudes y valores de respeto a la diversidad y no discriminación mediante el empleo de técnicas específicas, pero ello se debe completar con la existencia de un conjunto de normas que regulen estos aspectos de la convivencia.

\section{c) Organización y relaciones entre alumnos}

Relaciones entre alumnos, organización del aula, horarios, utilización de dependencias, comedor, entradas, salidas, patio, etc.

La vida en el aula y en el centro es muy intensa, en la medida que hay un grupo numeroso de personas que se ven obligados a convivir durante muchas horas al día en un recinto común. Ello hace necesario e imprescindible la existencia de un conjunto de normas que regulen estos aspectos de la convivencia. La falta de estas normas suele generar situaciones conflictivas y es una causa fundamental del deterioro del ambiente escolar.

\section{Cuidado y utilización de los materiales del aula y espacios comunes del centro}

Normas referidas al uso de los espacios y materiales que son de uso común, y que por lo tanto, al igual que todos los bienes públicos, requieren un cuidado especial para mantenerlos limpios y para evitar su deterioro.

El cuidado y utilización adecuada de los materiales comunes del aula, constituye una categoría claramente diferenciada de las demás, que con mayor o menor precisión suele estar regulada en la mayoría de los centros educativos. Junto a este aspecto, se ha incluido una segunda categoría con los espacios comunes del centro, dada la importancia que tiene para mantener un contexto agradable de trabajo y como precursor de la conservación de los bienes públicos en general.

También se establece una segunda categoría formada por espacios comunes del centro, dada la importancia que tiene para mantener un contexto agradable de trabajo y como precursor de la conservación de los bienes públicos, en general. Precisamente, el cuidado que se presta a estos espacios comunes es un indicador sólido del grado de organización del 
centro educativo. Cuando los profesores actúan de forma individual, ocupándose de lo que ocurre en «su» clase, pero desentendiéndose de lo que pasa de "puertas para fuera», el deterioro global del centro es inevitable. Por ello, el grupo de normas que regulan la utilización de estos espacios, tiene una importancia fundamental para la vida de un centro, y es necesario analizarlas de forma diferenciada.

\section{a) Materiales y objetos}

Materiales fungibles, mapas, esferas, libros de biblioteca, mobiliario, etc., que sea de uso común. Normas de uso y seguridad, eficacia, mantenimiento y economía, de los materiales del aula.

\section{b) Espacios comunes}

- Pasillos, servicios, escalera, patio, laboratorios, gimnasio, etc.

- Servicios: utilización de los mismos de modo adecuado, evitando el despilfarro de agua, papel higiénico, jabón, etc. así como el deterioro de las instalaciones.

- Escaleras: subidas y bajadas sin correr ni saltar, respetando a los alumnos más pequeños que las utilizan al mismo tiempo.

- Patio: Distribución de espacios por ciclos educativos o edades; uso de pistas deportivas y material, uso de las papeleras, fuentes, etc.

- Laboratorios y gimnasio: normas de uso y mantenimiento de los materiales existentes, tanto para la conservación de los mismos como para preservar la seguridad de los alumnos que los emplean.

- Comedor: entradas y salidas, comportamiento durante las comidas, recogida de la mesa, etc.

- Biblioteca: normas sobre préstamos, utilización de la sala de lectura, colocación de las sillas, etc.

\section{Trabajo en el aula}

Todas aquellas actividades individuales o colectivas que realizan los alumnos y que requieren una normativa que facilite la eficacia y calidad del trabajo realizado.

En los centros educativos constituyen un grupo de normas que se pueden considerar "particulares» de cada profesor, en la medida que cada uno suele tener sus normas de trabajo y tiene un forma de organizar los grupos o las responsabilidades en la clase.

En los planteamientos de la Reforma, muchos de estos aspectos pasan a ser contenidos actitudinales y, por lo tanto, susceptibles de programarse y coordinarse con el resto de los profesores, lo cual hace necesario un conjunto de normas unificadoras que los regulen.

Dentro de la gran variedad de trabajos y actividades que pueden realizar los alumnos en el aula, se han clasificado, atendiendo al número de alumnos que intervienen: individuales, en equipo y en gran grupo, al entender que cada una de ellas requiere unas normas específi- 
cas de funcionamiento. También se ha diferenciado la subcategoría «cumplimiento de responsabilidades», por constituir un tipo de actividad de alto nivel formativo, que cada vez adquiere más importancia en el medio escolar.

Las subcategorías de normas establecidas son las siguientes:

\section{a) Trabajos individuales}

Precisión, orden, rigor, claridad, limpieza, esfuerzo, temporalización, ayudas, etc.

Todos estos aspectos suelen estar regulados de manera explícita o implícita y forman parte del nivel de exigencia de cada profesor. Cuando existe coordinación entre los profesores que intervienen en un mismo grupo de alumnos, podemos hablar de un grupo de normas comunes que al cabo del tiempo pasan a formas parte de la cultura organizativa del centro, en la medida que se consolidan y llegan a ser asumidas por los alumnos.

\section{b) Trabajos en equipo}

Planificación y realización de experiencias, juegos, coordinación, aportación de ideas, diálogo, participación responsable, respeto al encargado de dirigir el grupo etc.

Constituyen un grupo de normas que puede ser más o menos amplio, según el número de actividades comunes que se realicen habitualmente en el aula. Por ello, dependen mucho del modelo de enseñanza que se adopte en un centro o en el equipo docente.

\section{c) Actividades en gran grupo}

Actividades deportivas, representaciones, audiciones, conmemoraciones, etc. Son actividades en las que participan todos los alumnos de un aula, curso, ciclo, etapa educativa o incluso todo el centro escolar. El desarrollo satisfactorio de estas actividades depende en gran medida de la existencia de un conjunto de normas, y de que éstas sean puestas en práctica de manera rigurosa por parte del profesorado del centro.

\section{d) Cumplimiento de responsabilidades}

Jefe de equipo, secretario, delegado de clase, encargado de material, encargado de biblioteca, encargado de las plantas, etc. Cada actividad está regulada por una norma que especifica lo que deben hacer los alumnos encargados de llevarla a cabo.

\section{Educación para la salud}

Hace referencia a todas aquellas situaciones y actividades, de carácter individual o colectivo, que afectan directamente a nuestra salud y bienestar. 
Constituyen un grupo de normas claramente diferenciado, que aborda un tema educativo fundamental, y que la Reforma plantea como contenido actitudinal dentro de los diversos bloques temáticos y como tema transversal.

La distinción de dos subcategorías se hace para facilitar el análisis de las normas de este grupo, al entender que en las primeras edades deben predominar más las normas de higiene $\mathrm{y}$ aseo personal, para pasar progresivamente a las del cuidado del cuerpo.

Las subcategorías de normas establecidas son las siguientes:

\section{a) Cuidado del cuerpo}

Aspecto personal cuidado, alimentación sana, prevención de enfermedades, prevención del consumo de drogas, prácticas deportivas, conductas posturales, salud, etc. Son aspectos, todos ellos, que se trabajan como contenidos curriculares de tipo actitudinal, pero requieren el soporte de un grupo de normas que generen hábitos positivos.

\section{b) Higiene y aseo personal}

Hábitos de limpieza, hábitos higiénicos, prevención de parásitos, prevención de infecciones, etc. el medio escolar es especialmente adecuado para crear en los alumnos hábitos de higiene y aseo, a través del aprendizaje de normas.

\section{Comportamiento como ciudadano en el ámbito extraescolar}

Se refiere a aquellas situaciones en las que se realizan excursiones a espacios naturales, parques, plazas, edificios históricos, museos, iglesias, bibliotecas, etc., y que requieren un adecuado comportamiento de los alumnos y la adopción de una especial sensibilidad por la conservación de estos lugares.

Forman un grupo de normas muy específico que regulan el comportamiento y aprendizaje de los alumnos, mayoritariamente, fuera del centro educativo, donde se dan ocasiones muy adecuadas para aprender a respetar y conservar los lugares visitados, y a ser capaces de trasladar estas actitudes positivas a otros contextos.

Se han diferenciado tres subcategorías de normas, para poder hacer un análisis más pormenorizado de las normas que incluye la LOGSE sobre estos aspectos:

\section{a) Recursos y espacios naturales}

Utilización adecuada del agua y la electricidad, evitando despilfarros innecesarios. Excursiones a zonas de montaña, playa, parques naturales, etc. Uso adecuado de los lugares visitados, evitando el deterioro o la suciedad. Protección del medio ambiente. 


\section{b) Lugares públicos}

Excursiones a parques públicos, plazas, fábricas, teatros, edificios, etc. Requieren la existencia de unas normas muy específicas que aseguren, tanto el respeto a los lugares visitados, como la propia seguridad de los alumnos.

\section{c) Patrimonio histórico-artístico}

Visitas a ruinas históricas, excavaciones arqueológicas, monumentos, museos, edificios históricos, pinacotecas, representaciones, películas, etc. Para valorar y preservar este patrimonio, es necesario que los alumnos se comporten de acuerdo con las normas establecidas, las cuales, dadas las características de los lugares visitados, son mucho más rígidas que en otras situaciones educativas.

\section{Referencias bibliográficas}

Curwin, R. L. y Mendler A. N. (1987): La disciplina en clase. Madrid. Narcea.

Hargreaves, D. H. (1975): Interpersonal relations and Education. Edición revisada, Routledge and Kegan Paul.

Krings, H. (1978): Conceptos fundamentales de filosofia. Tomo II. Barcelona. Herder.

Parsons, T. (1951): The Social System. Glencoe, Ill. Free Press.

Pepper, C. (1960): Ethics. New York. Appleton.

Pérez, C, (1995): Las normas en la LOGSE. Aprendizaje de normas en al aula mediante la participación democrática. Tesis Doctoral, Universitat de Valencia.

Schmuck, R. A. y Schmuck, P. A. (1971): Group processes in the classroom, Dubuque, Iowa. C. Brown Company Pub. (Traducción castellano: Técnicas de grupo en la enseñanza, México, pax)

Turiel, E. (1984): El desarrollo del conocimiento social. Moralidad y convención. Madrid: Debate. Von Wright, G. (1970): Norma y acción. Madrid. Técnos.

Willians, R. S. (1975): «Normas» en Enciclopedia Internacional de las Ciencias Sociales, vol 7. Madrid. Aguilar.

Fecha de recepción: 16-XI-00

Fecha de revisión: 3-V-01

Fecha de aceptación: 16-X-01 


\section{ANEXO}

\section{Categorización de las normas escolares}

a) Normas que regulan la convivencia en el aula y en el centro

1. Organización y relaciones entre los alumnos

2. Respeto a la diversidad, no discriminación

3. Derechos y deberes de los alumnos

4. Otras normas

b) Normas referidas al funcionamiento democrático del aula

1. Participación en el establecimiento de normas

2. Asambleas, debates, reuniones, etc.

3. Respeto a las decisiones tomas democráticamente

4. Otras normas

c) Normas que regulan el cuidado de los materiales

1. Materiales del aula

2. Materiales comunes del centro

3. Otras normas

d) Normas que regulan la realización de tareas en el aula

1. Trabajos individuales

2. Trabajos en equipo

3. Actividades en gran grupo

4. Otras normas

e) Normas que regulan las salidas del centro

1. Cuidado de los recursos y espacios naturales

2. Cuidado de los lugares públicos

3. Cuidado del patrimonio histórico-artístico

4. Otras normas

f) Otras categorías de normas

1.

2.

3. 